\title{
Investigation of environmental influences on wireless localization techniques for outdoor applications
}

\author{
Max Uhlig' ${ }^{1}$, Dr. Thomas Keutel ${ }^{1}$, Prof. Olfa Kanoun ${ }^{1}$ \\ ${ }^{1}$ Chemnitz University of Technology, Chair of Measurement and Sensor Technology, Germany \\ max.uhlig@etit.tu-chemnitz.de, thomas.keutel@etit.tu-chemnitz.de, olfa.kanoun@etit.tu-chemnitz.de
}

\begin{abstract}
:
Repeatedly, there are many victims caused by landslides. To warn the people for a threatening landslide we developing a monitoring system for unstable slopes, dikes and shores. The measure system based on autonomous, wireless sensor nodes. These nodes forming a grid in which the distances between the nodes will be observed continuously. In case of any instability, the system is able to detect minimal ground movements and can send an emergency signal to a geographic information system to warn the competent authorities. The main challenges of the development process are first the realization of a self-organized power supply and second the determination of environmental influences for the outdoor localization witch will be discussed within this contribution.
\end{abstract}

Key words: monitoring system, wireless sensor nodes, outdoor localization, distance measurement.

\section{Introduction}

Wireless sensor networks hold the promise of many new applications in the area of condition monitoring and control. Examples include target tracking, intrusion detection, wildlife habitat monitoring, climate control, and disaster management. The underlying technology that drives the emergence of sensor applications is the rapid development in the integration of digital circuitry, which will bring us small, cheap, autonomous sensor nodes in the near future. [1] We developing an autonomous sensor node which is able to detect minimal shifts from unstable slopes, dikes or shores if the node works in a universal grid. The information of such shifts can be used to warn people for a threatening landslide.

\begin{abstract}
Application scenario
Established techniques like optical fibers or geotextiles who inserted into the ground are expensive. Furthermore it is not even possible to insert these materials in a dangerous environment or in a nature reserve. Our aim is to develop a low cost, easy to install, selforganizing and autonomous working sensor grid for slopes, dikes or shores monitoring. Each node in this grid has the task to measure the distance between oneself and the other nodes in the grid continuously. Is there a deviation in the results of the measurements we have an indicator for a movement which we can report over a local gateway to a geographic information system. To do this a very accurate and robust measure system is required.
\end{abstract}

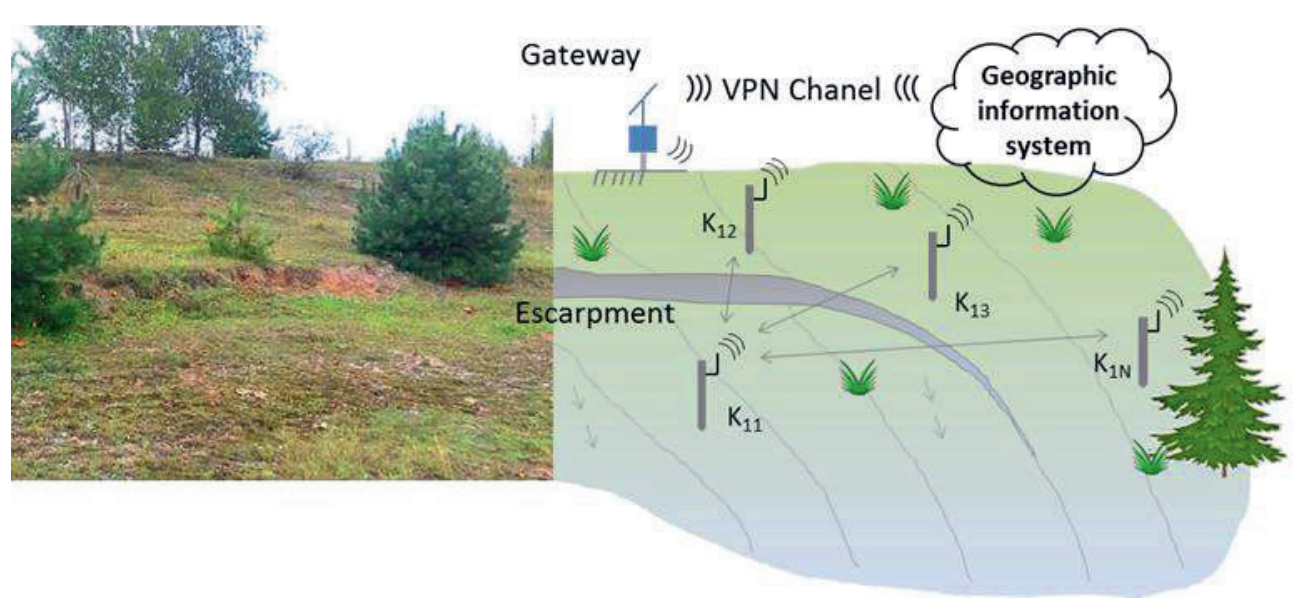

Figure 1: schematic of the randomly placed sensor nodes for slope monitoring 


\section{Method}

To compliance the high energy restrictions from the autonomous sensor nodes, we use a high precision, high efficient ranging system from decaWave. The system use a technique called "time of flight" to determine the distances between each measure point. The ranging system is developed for indoor usage. Therefore we have to investigate the outdoor environment influences like changing temperature, humidity, rain, fog and so on.
To do that we prepared an experimental field which allows us to log relevant disturbance variables directly in the propagation path. We did long time measurements as a part of our verifications to characterize the impact of these factors of the accurateness of the measured distances. The following plot illustrate the temperature and the humidity impact on the measured distances between two fix points.
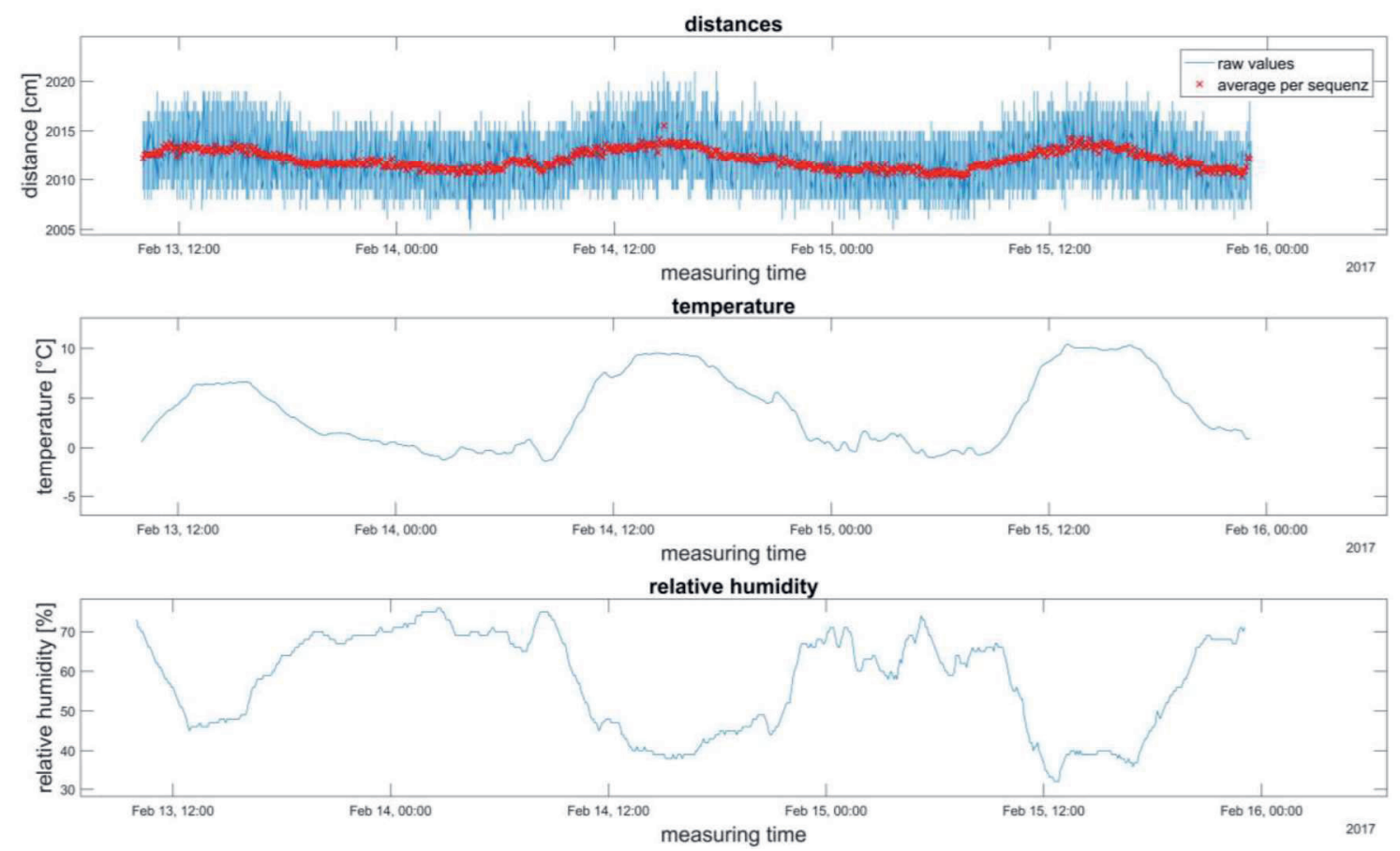

Figure 2: temperature and humidity impact on distance measurements

To get stable values and for reducing noise we did one measure sequence every 5 minutes. Every sequence consist of 50 fast single distance measurements. Based on this values we calculated an average visualized as the red crosses.

It becomes clear, that changes in temperature and humidity are correlated with deviations in the distance measurements. We combine these outcomes with information about other potential disturbance variables to produce an algorithm which is able to compensate the outdoor environment influences. As a part from our monitoring system this algorithm allows us to calculate more stable and accurate values.

Within the final contributions further aspects of the setup, the experiments and further influences of the outdoor localization issue will be discussed and presented.

\section{References}

[1] K. Langendoen *, N. Reijers (2003), Journal Elsevier Computer Networks, doi:10.1016/S13891286(03)00356-6

[2] Mao, G., \& Fidan, B. (2009). Localization algorithms and strategies for wireless sensor networks. Hershey: Information Science Reference. ISBN: 9781605663968

[3] Zekavat, R., \& Buehrer, R. M. (c2012). Handbook of position location: Theory, practice, and advances. Hoboken, NJ: Wiley [u.a.]. ISBN: 9780470943427 